\title{
MAKING MANUFACTURING CHANGES LESS DISRUPTIVE: AGENT-DRIVEN INTEGRATION
}

\author{
Joseph Neelamkavil, \\ Weiming Shen, Qi Hao, Helen Xie \\ Integrated Manufacturing Technologies Institute \\ National Research Council Canada \\ joseph.neelamkavil@nrc.gc.ca; \\ weiming.shen nrc.gc.ca; qi.hao nrc.gc.ca; helen.xie nrc.gc.ca
}

\begin{abstract}
This paper presents some results of our recent investigation on how to address changes in mamufacturing environments. The results presented here are based on our recent visits to manufacturing plants (in order to understand current industrial practice) and a comprehensive literature review. It has become apparent that agents and multi-agents based technologies have the power and flexibility to deal with the shop dynamics. This paper also discusses research opportunities and challenges in this area, presents our recent research work in developing agent-based technologies to streamline and coordinate design and production activities within a manufacturing enterprise, and between the enterprise and its suppliers.
\end{abstract}

\section{INTRODUCTION}

In recent years manufacturing companies have gone through a rapid paradigm change. This change is primarily due to a shift from vendor's market to that of customer's. Customers want products with lower prices, higher quality and faster delivery; yet they also demand products customized to match their unique needs. The customer demands also fluctuate over short periods of time; yet if not served promptly, they are lost to the competition; products also become obsolescent in a short time for a variety of reasons (out of fashion, new products in market, energy cost, and so on). To meet with many of these challenges, manufacturers will require a paradigm shift to adapt their business model to be compatible with agility and mass customization. It combines mass production's economies of scale with custom manufacturing's flexibility. Requirement for lower product cost has dictated reductions in production cost also, which further causes a ripple effect on the complete production process, and demand enhanced productivity and flexibility. But the productivity targets get bypassed when unexpected changes (described as disturbances often) occur at the shop level (e.g., new production/process introduced, 
machine breaks down, parts missing, etc.). For most manufacturers, it is a challenge to deal with the frequent product changes and customer demand fluctuations.

It should be stated that, the changes attributable to order fluctuations, cancellations and occasional wrong decisions are unavoidable in an industry. For example, to realize an undesired design decision would trigger changes to products data. The impact of the changes needs to be assessed properly, and the focus in this research is to study how best the impact of both planned and unplanned changes can be addressed at the manufacturing shop and resource level, to optimize production via downtimes reduction and optimum utilization of the plant resources, within the constraints imposed by the factory dynamics. Rapid reconfigurations of the manufacturing, flexible planning, and fast response control systems have been echoed as the business models to deal with these scenarios. To crystallize our ideas above, we visited several manufacturing plants, recorded and analyzed findings, classified and summarized the results, and arrived at approaches that could deal with the changes and disturbances in production - the changes - in general.

This paper presents some results of our recent investigation on how to address changes in manufacturing environments. The results presented here are based on our recent visits to manufacturing plants (in order to understand current industrial practice) and a comprehensive literature review. It has become apparent that agents and multi-agents based technologies have the power and flexibility to deal with the shop dynamics. This paper also discusses research opportunities and challenges in this area, and briefly presents our recent research work in developing agent-based technologies to streamline and coordinate design and production activities within a manufacturing enterprise, and between the enterprise and its suppliers.

\section{EFFECT OF CHANGES ON PRODUCTION}

In a manufacturing shop, the occurrence of changes is a daily event. The term "change" here is used to mean those, which occurs primarily at the production level. It is somewhat different from the management of engineering changes (often termed as Engineering Change Management - ECM), which is a well-established area. The ECM is meant to cater for the changing needs of products and product functionalities; and more details of this topic can be found in (Pikosz and Malmqvist, 1998). In the manufacturing context, the major reasons why changes are bound to occur are the following:

- Customers change their minds and come up with new requirements

- Suppliers stumbles on their delivery status

- Customer service collects recalls and expects quick fixes

- Design engineers improve functionality and introduce new product variants

- Marketing \& Sales adds extra features and likes introducing newer products

- Production implements continuous improvement and new product lines

- Unexpected breakdown creates chaos on the shop floor

- Manufacturing adds new production techniques

- Regulation forces change both at the product and process levels.

The MASCADA report (Valckenaers, 1998) has come up with distinct definitions for the terms production "changes" and production "disturbances", namely: "A production change is an alteration to the production conditions, which is 
intentionally performed by the plant. A production disturbance is an unanticipated change to production conditions with a negative effect on the (manufacturing) process performance". The important characteristic that distinguishes changes from disturbances is pivoted on the question whether the production facility intends the change. Bear in mind that, a disturbance in one area of production can cause a change in another area, and vice versa. Looking differently, a production change is a planned event, while a disturbance is something that is not at all planned, and hence can only be reacted to.

From a research viewpoint, the impact of changes and disturbances is often assessed from a qualitative perspective. Note that, a quantitative assessment is difficult to attain because, the real impact can vary from plant to plant, and location to location. The nature of disturbance also plays an important role - whether these are caused by external factors (e.g., customers and suppliers) or internal matters (e.g., machine breakdown). The effect of disturbance based on internal matters also depends on criticality of the resources involved (e.g., a key machine), or whether it is localized or global within the plant (e.g., a power outage in the plant).

To conduct research on impact of changes, firstly one has to collect data pertaining to the changes and disturbances; the reliability and the source of the data also will become critical. For example, a senior executive in a plant may not even be aware of the machine break down of a manufacturing cell. The noted MASCADA study (Valckenaers, 1998) based their findings on 19 plants from 17 European companies (13 being discrete manufacturing facilities) categorized the disturbances into 4 categories, based on who/what caused them: a) customers, b) suppliers, c) internal (e.g., resource, quality or co-ordination breakdown) and d) regulatory/environmental. Also, the disturbances may be abrupt vs. gradual (e.g., wear \& tear), random vs. systematic (e.g., avoid production during peak electrical hours), time (e.g., late delivery) vs, poor quality based. The above study has categorized the changes into 4 categories also: a) introduction of new products or product variants, b) increase/decrease in production capacity, c) introduction of new production technology, and d) changes in work force.

In most plants studied by the MASCADA team, it was discovered that most planning and control systems treated disturbances (sometimes the changes also) as exceptions that were undesirable, and were therefore ignored mostly during regular planning activities. The planning systems were based on a top-down approach (MRP, for example), which didn't allow any feed back from the lower levels. This means that if production gets deviated from the plan, the planning needs to be restarted again. The study concluded with a number of guidelines on how to handle the changes and disturbances:

- Manufacturing systems need to be easily adaptable to new requirements.

- Manufacturing systems need to be scaleable at small steps.

- Workers and engineers need to be integrated seamlessly into the production process that optimizes the usage of technical and human resources.

- Shop systems need to be able to flexibly react to varying production orders.

- Products and production processes need to be structured such that the product distinguishing features are built-in as late as possible.

- Shop processes should be organized in such a way that if disturbances do occur, production can continue with reduced capacity instead of just collapsing it. 
- If disturbances are bound to occur, resources need to be re-assigned efficiently. The re-assignment however, should not cause any more disturbances.

- Planning and control systems should make global consequences of local decisions explicit to user, and support the co-ordination of all related decisions.

\section{INDUSTRY PRACTICE}

We visited several manufacturing plants (truck manufacturing plant, medical instrument manufacturer, landing gear assembly plant, mold making and injection molding shops, etc.) to discuss the impact of changes in production, and to find out issues and problems that typically exist between the design and production departments - like integration issues, data handling issues, change management issues, communication issues, and so on. Some of the findings are the following:

The need to deal with change is woven all over onto the truck manufacturing operations. The visited plant assembles over 100 trucks daily, and operates 3 shifts/day, having variable production rates for each shift. It is a challenge to address the schedule change issues, and to do proper load balancing since the chassis line, engine line, cab line, etc. need to converge to proper entry points just in time for assembly to take place without hampering the production line. In addition, every second or third truck is of a totally different kind, and the changes and variations due to different parts combination become large. The plant needs to deal with over 10,000 different kinds of parts; and the occurrences of missing (or wrong) parts or parts not at right place at right time become a common event. In some manner, the assembly line and the material management system have to absorb such changes, or come up with provision to complete the assembly offline at some other time. In trucking industry, they have a relatively long (3-5 year time frame) product development time.

The medical instrument manufacturing plant that we visited focuses on the design and assembly of the instrument, while the actual manufacturing is outsourced. Their product development time is relatively short (1 to 1.5 year time frame). Requirement changes, missing and/or incomplete requirements have been problem areas. The product development cycle needs to cater for prototyping and piloting prior to full production. Integration and traceability issues throughout the complete product life cycle are seen very important, and are brought under control via frequent but formalized stage gating procedures. This is a time consuming process, but it works for them, as there are not many players manufacturing the same kind of apparatus. Material management is well under control as they have limited number of parts to deal with, while at the same time they follow a strict lean manufacturing and part replenishment procedures.

The issues associated with the changes in production and integration problems in other plants fall typically somewhere between the two cases described above, and they are not elaborated here. 


\section{SITUATION ANALYSIS}

Based on the industry visits that we have had so far, the two most important issues that became noticeable were 'embracing the change' and the 'need for integration'. In this context and throughout this research study, the phrase 'change' is used loosely to mean both the 'changes' and the 'disturbances'. We observed that, the effects of changes in industries have been addressed mostly at the business and information technology level only. Not much attention is given to the changes of the manufacturing system itself; yet the shop floor suffers from continuous evolution of changes that are imposed on it.

It appears that the reasons why industries find it hard to cope with a change is due to inefficient communication procedures, ineffective shop floor control systems, and the difficulty of achieving integration arising out of several technology components and islands of automation. For example, a capacity analysis software determines a master production schedule that sets long-term production targets; a scheduling system determines the sequence in which manufacturing resources are used in producing the products; a manufacturing execution system tracks the realtime status of work in progress, enforces process plans, and reports labor/material claims. In addition, these applications are often legacy systems developed over a number of years. Each system may perform well for its designated tasks; they need to respond very quickly to changes, but often are not equipped well to handle complex business decisions that involve the coordination of several applications. Rapid but timely solutions to these scenarios are crucial to agile manufacturing, especially in the current era of globalization, automation, and telecommunication.

Recalling what we mentioned earlier, shorter product life cycles and global competition have forced many manufacturing companies to become agile. Industry needs to be able to respond very quickly to deal with this and to minimize the impact of changes on production performance. In this environment, configuring the shops for production and establishing effective control is crucial. Valckenaers et al. (1999) have summarized the requirements associated with this scenario into 5 categories namely: proactive-ness, reactivity, flexibility, reconfigure-ability and connectivity.

By being proactive, a shop control system can plan for the future. It anticipates certain events, and is guided to those states in which such events are best handled, if they do occur. Proactive-ness also enables a shop control system to look ahead into the future. By being reactive, the control system is able to react rapidly to certain events and adapt to such situations. Alternative shop resources, transportation routes, process plans and trade-offs between yields and speeds are examples of process flexibility. The user interaction flexibility addresses how flexible a system supports a specific user to perform several tasks, or how many users can do certain tasks. Reconfigure-ability is a feature that allows for easy adaptation of the system for new productions, product variants, plant layouts, and so on. How tightly a shop control is connected to an actual production environment and its equipment controllers are representative of the connectivity. Included here is the ability to collect data from factory floor to perform the required shop floor control. The control system maintains and has access to the latest available information about shop resources, and is able assign/reassign orders to any available resources.

According to Wyns (1999), a shop floor control is that group of activities directly responsible for managing the transformation of a planned order into a set of 
outputs, and those indirect activities that needs to be done to cover the changes or adaptations of the control solution in an easy way. During the operative phase, it governs short-term detailed planning, execution, and monitoring of process preparation and resource allocation activities that are needed to control the flow of work order from the time it is released by planning system for the execution, until the order is completed. Two well-recognized shop floor reference architectures that are much contextual here are the 'holonic' manufacturing and multi-agent manufacturing systems (Deen, 2003; Marik et al., 2003; Shen et al., 2001; van Brussel et al., 1998).

\section{CHALLENGES AND OPPORTUNITIES}

Based on the industry practice analysis and research literature review, a number of research challenges as well as opportunities have been identified.

\section{System Architecture}

To create a system that supports agile shop floor control is a hard and complex task. The challenge here is to come up with an architecture that supports agility, and that facilitates the design of control systems that accommodates heterogeneous manufacturing resources, yet without expending too much programming effort. It requires the formulation of an instant manufacturing cell (Barata de Oliveira, 2003) (with various rules and procedures to work in harmony) composed of various resources represented by multi-agents, based on new and/or changed capacity. Issues related to the integration of several controllers, as well as their co-ordination need to be supported.

\section{Modeling and Presentation of Agents in Action}

A major thrust here is to formulate a modeling paradigm that can address both the modeling and controlling aspects. Creation of agent models that are representative of all shop resources with pertinent capability features and control aspects is always a challenge. It should be dynamic in the sense that any modifications and/or capability changes to the physical resource should reflect in its agent model immediately. At the same time one has to ensure that it doesn't overreact to minor changes (like spending more time changing its mind rather than acting, or triggering excessive setups and material handling activities in lieu of production). For easy communication with the manufacturing personnel, an agent embedded system should also provide good presentation and visualization capabilities, perhaps with the aid of virtual models.

\section{Ontology-based Communication}

At a high level, ontology aims at sharing and reusing knowledge by providing an explicit specification of conceptualization. It can support agent communications in two ways. First, it can provide common vocabulary for communication and shared understanding. Second, it can support data interoperability among agents or 
computer systems by providing semantic foundations for translators. Consider the scenario in which a planning agent wants to communicate with a resource agent regarding various machine tools. In particular, a terminology they may need to communicate is the name of machine tool manufacturer. In a new design of agents, one can take advantage of ontology by specifying a common vocabulary, i.e. "Made by" for the machine tool manufacturer. And, agents can use this common term for communication. In a legacy design, the planning agent and resource agent may regard the machine tool manufacturer as "Manufactured by" and "Maker is", respectively. Ontology can provide semantic foundations by mapping "Maker is" and "Manufactured by" to "Made by", enabling agent interactions in natural manner.

\section{Data Collection \& Filtering}

The systems environment should be developed based on a data-centric approach in which sensors couple with databases that ensures quality, integrity, reliability, redundancy and security. In a dynamic shop environment, on-line and automatic data collection from the physical shop floor will become almost mandatory. A form of data mining and filtering is necessary to screen out noise and other unnecessary data. If decisions are taken based on real-time simulations, then to ensure selection and extraction of correct data towards on-line simulation always poses a challenge. Data required for real-time decisions, and the amount of details (hence the data) that need to be modeled will be very critical in a simulation based control approach.

\section{Quality of Decisions and Real-time Adaptation}

In an agile environment, the shop dynamics will necessitate a manufacturer to deal with frequent changes, and real-time simulation will be helpful to try out different scenarios, and to make decisions based on an optimized shop. Artificial intelligence and distributed simulation technologies can be applied to address such real-time decision processes, which can also help to reduce the computation time involved in making those decisions (Yoon and Shen, 2005). The quality of decision itself is affected by factors such as the simulation frequency, embedded details, incorporated assumptions, and so on.

\section{Conflict Resolution}

In the shop environment the resources always face the dilemma of local vs. global optimization. Reconciling different viewpoints and conflicting objectives among resources (hence agents trying to coordinate their actions) is a real shop floor challenge. The agents need to act intelligently in making decisions and taking actions while at the same time accommodating the global effects of local decisions.

\section{AGENT-BASED SOLUTIONS}

In the change scenario, a manufacturer needs to be able to implement, execute, update, and maintain the network representing the launch of new products and/or 
processes, and also addresses those issues pertaining to changes that are forced upon on the shop floor. Delays and failures may affect the production processes and transportation system, which may force re-grouping or re-routing of the orders. When re-routing or re-scheduling is needed, one has to ensure that the material handling aspects are also taken care of. A tool built to tackle such issues must support the user in coordinating and controlling most of these activities through a set of small, distributed, autonomous, configurable, intelligent, and communicating systems designed to satisfy their specific goals, while globally achieving optimum performance at the enterprise level (Paolucci and Sacile, 2005). It is becoming somewhat obvious that multi-agent systems (Peng et al., 1999; Brückner et al., 1998; Shen and Norrie, 1999; Parunak, 1999) provide promising technologies for rendering the modern shop floor to be agile.

The manufacturing resources, typically under the control by various controllers, are the basic set based on which the complete factory production is planned. Each resource (representative of an agent) has its own basic core capabilities or core skills, and through cooperation can build additional capabilities. And when they interact and cooperate, they can generate aggregated functionalities that are compositions of their individual capabilities. Many resources need to work together in harmony to deal with the changes and to achieve a common goal. For the manufacturing system to work well within the dynamics of production, an agentbased system needs to know what constitutes a change upfront. With help from a human, or instruction from an analysis support module, a decision module can decide on what constitutes a change. A control module can decide on what data is to be monitored to detect those changes. Based on messages from the control module on the occurrence of a change, the decision module can make contextual decisions, in liaison with the support module. Here, the breakdown of a critical machine can be considered as a big change, whereas failure of other machines may be considered a small one. Proper coordination and evaluation of the resource capabilities are needed to deal with the change scenario. Essentially, one can think of the change scenario in terms of a manufacturing resource configuration and allocation problem in real-time.

Based on our understanding of industrial needs and our experience on agentbased collaborative design and manufacturing, particularly agent-based manufacturing scheduling and as described in (Shen et al., 2005; Hao et al., 2005; Shen and Norrie, 2001), we are currently working in collaboration with industrial partners on following research directions:

- Implementing just-in-time materials handling using intelligent software agents and optimization techniques. Some results of this research work are reported through a separate paper submitted to the same conference by Hao and Shen (2006).

- Investigating schedule repair and buffer size optimization through agent-based simulation.

- Integrating RFID (Radio Frequency Identification) technology with agent-based real-time shop floor scheduling and materials handling.

- Developing software tools by integrating software agents and workflow technology for coordinating/managing between design and production departments. 


\section{CONCLUDING REMARKS}

Shorter product life cycles and global competition have forced manufacturing companies to become very dynamic and agile. Modern manufacturing environments are subject to frequent and rapid changes (e.g. new products or product variants, machine breakdowns, demand fluctuations, etc.). Manufacturers need to respond quickly to deal with this situation in order to minimize the impact of changes on their production performance, and for the survival of the organizations. Agent technology and multi-agent systems have the power and flexibility to provide rapid assessments on the need for production changes and factory dynamics, recommend and/or trigger real-time actions, and adjust manufacturing plans and production.

Our broad vision is to develop agent-based technologies to streamline and coordinate design and production activities within a manufacturing enterprise, and between the enterprise and its suppliers. We believe that, by integrating with other advanced technologies including cooperative workflow, simulation, and optimization, intelligent software agents can be successfully applied to address dynamic changes in the manufacturing shop floor locally without disruptions to the overall production - a problem that cannot be solved by traditional planning and scheduling systems because these changes are not predictable in advance.

\section{REFERENCES}

1. Barata de Oliveira, JA. "Coalition Based Approach for Shop Floor Agility - A Multi-agent Approach", Ph D Thesis, University of Lisbon, Portugal, 2003.

2. Brückner, S, Wyns, J, Peeters, P, Kollingbaum., M. "Designing Agents for Manufacturing Control", 2nd AI \& Manufacturing Research Planning Workshop. Albuquerque, NM. August 1998.

3. Deen, SM (Ed.) Agent-Based Manufacturing - Advances in the Holonic Approach. Springer-Verlag, Heidelberg, Germany, 2003.

4. Hao, Q, Shen, W. "An Agent-Based Simulation of a JIT Material Handling System". Submitted to BASYS'06, 2006.

5. Hao, Q, Shen, W, Zhang, Z. An Autonomous Agent Development Environment for Engineering Applications, International Journal of Advanced Engineering Informatics, 2005, 19(2):123-134.

6. Marik, V, McFarlane, D, Valckenaers, P. (Eds.) Holonic and Multi-Agent Systems for Manufacturing. Springer-Verlag, Heidelberg, Germany, 2003.

7. Paolucci, M, Sacile, R. Agent-Based Manufacturing and Control Systems, CRC Press, New York, 2005.

8. Parunak, HVD. "Industrial and Practical Applications of DAI". In Multi-agent System: a Modern Approach to Distributed Artificial Intelligence, G. Weiss (Ed.), Cambridge, Massachusetts: MIT Press, 1999, pp. 377-421.

9. Peng, Y, Finin, T, Labrou, Y, Cost, RS, Chu, B, Long, J, Tolone, WJ, Boughannam, A. "An AgentBased Approach for Manufacturing Integration - The CIIMPLEX Experience", International Journal of Applied AI, 1999, 13(1-2):39-44.

10. Pikosz, P, Malmqvist, $J$. "A comparative study of engineering change Management in three Swedish engineering Companies”, DETC98, Paper No DETC98/EIM-5684, Atlanta, GA, USA, 1998.

11. Shen, W, Norrie, DH. "Agent-Based Systems for Intelligent Manufacturing: A State-of-the-Art Survey", Knowledge and Information Systems, an International Journal, 1999, 1(2):129-156.

12. Shen, W, Norrie, DH. "Dynamic Manufacturing Scheduling Using both Functional and Resource Related Agents". Integrated Computer-Aided Engineeting, 2001, 8(1):17-30.

13. Shen, W, Norrie, DH, Barthes, J-P. Multi-Agent Systems for Concurrent Intelligent Design and Manufacturing, Taylor and Francis, London, UK, 2001.

14. Shen, W, Lang, S, and Wang, L. iShopFloor: An Internet-Enabled Agent-Based Intelligent Shop, IEEE Transactions on Systems, Man, and Cybernetics, Part C, 2005, 35(3):371-381. 
15. Valckenaers, P. "WP1 Dissemination Report: Analysis and evaluation of change and disturbances in industrial plants (MASCADA ESPRIT LTR 22728)," tech. rep., K.U. Leuven PMA, A.I. Systems, Daimler Benz Research, University of Cambridge, VTT Automation, 1998.

16. Valckenaers, $\mathrm{P}$, Heikkilä, $\mathrm{T}$, Baumgaertel, $\mathrm{H}$, McFarlane, D, Courtois, J-P. "Towards a novel manufacturing control principle", Second International Workshop on Intelligent Manufacturing Systems, Katholieke Universiteit Leuven, 1999, pp. 871-875

17. van Brussel, H, Wyns, J, Valckenaers, P, Bongaerts, L, Peeters, P. "Reference Architecture for holonic Manufacturing Systems: PROSA". Computers in Industry, 1998, 37(3):255-274.

18. Wyns, J. "Reference Architecture for Holonic Manufacture - the key to support evolution and Reconfiguration". Unpublished PhD thesis, Katholieke Universiteit Leuven, Leuven, 1999.

19. Yoon, HJ, Shen, W. "Simulation-Based Real-Time Decision Making for Manufacturing Automation Systems: A Review”, Int. J. Manufacturing Technology and Management, 2005. 